\title{
Generalized Hasimoto Transform of One-Dimensional Dispersive Flows into Compact Riemann Surfaces
}

\section{Eiji ONODERA}

Mathematical Institute, Tohoku University, Sendai 980-8578, Japan

E-mail:sa3m09@math.tohoku.ac.jp

Received December 18, 2007, in final form May 14, 2008; Published online May 20, 2008

Original article is available at http://www.emis.de/journals/SIGMA/2008/044/

\begin{abstract}
We study the structure of differential equations of one-dimensional dispersive flows into compact Riemann surfaces. These equations geometrically generalize two-sphere valued systems modeling the motion of vortex filament. We define a generalized Hasimoto transform by constructing a good moving frame, and reduce the equation with values in the induced bundle to a complex valued equation which is easy to handle. We also discuss the relationship between our reduction and the theory of linear dispersive partial differential equations.
\end{abstract}

Key words: dispersive flow; Schrödinger map; geometric analysis; moving frame; Hasimoto transform; vortex filament

2000 Mathematics Subject Classification: 35Q55; 35Q35; 53Z05

\section{Introduction}

We study the structure of dispersive partial differential equations of the form

$$
\begin{aligned}
& u_{t}=a \nabla_{x}^{2} u_{x}+J_{u} \nabla_{x} u_{x}+b g_{u}\left(u_{x}, u_{x}\right) u_{x}, \\
& u_{t}=-a J_{u} \nabla_{x}^{3} u_{x}+\left\{1+b g_{u}\left(u_{x}, u_{x}\right)\right\} J_{u} \nabla_{x} u_{x}+c g_{u}\left(\nabla_{x} u_{x}, u_{x}\right) J_{u} u_{x},
\end{aligned}
$$

where $N$ is a Riemann surface with an almost complex structure $J$ and a hermitian metric $g, a, b, c \in \mathbb{R}$ are constants, $u=u(t, x)$ is an $N$-valued unknown function of $(t, x) \in \mathbb{R} \times \mathbb{R}$, $u_{t}(t, x)=d u_{(t, x)}\left((\partial / \partial t)_{(t, x)}\right), u_{x}(t, x)=d u_{(t, x)}\left((\partial / \partial x)_{(t, x)}\right), d u_{(t, x)}: T_{(t, x)} \mathbb{R}^{2} \rightarrow T_{u(t, x)} N$ is the differential of the mapping $u$ at $(t, x), \nabla_{x}$ is the covariant derivative with respect to $x$ along the mapping $u$, and $J_{u}$ and $g_{u}$ denote the almost complex structure and the metric at $u \in N$ respectively. Recall that an almost complex structure $J$ on $N$ is a bundle automorphism of the tangent bundle $T N$ satisfying $J^{2}=-I$. Note that (1) and (2) are equations for vector fields along the mapping $u$.

In mathematics, a non-Kowalewskian-type evolution equation is said to be "dispersive" if its initial value problem is (expected to be) well-posed in both directions of the past and the future. See [10, Lecture VII]. Loosely speaking, in classical mechanics, a linear evolution equation with constant coefficients is called "dispersive" if the propagation speed of its plane wave solutions changes in proportion to the size of frequency. Typical examples of dispersive partial differential equations are the Schrödinger evolution equation of free particle $v_{t}+i v_{x x}=0, i=\sqrt{-1}$ and the Korteweg-de Vries equation $v_{t}+v_{x x x}-6 v v_{x}=0$. We call the solution of (1) or (2) a dispersive flow. In particular, when $a=b=0$, the solution of (1) is called a one-dimensional Schrödinger map.

Dispersive flows arise in classical mechanics: the motion of vortex filament, the Heisenberg ferromagnetic spin chain and etc. See $[3,4,5,6,7,16]$ and references therein. Solutions to such equations in classical mechanics are valued in two-dimensional unit sphere. The equation (1) is the geometric generalization of equations proposed by Fukumoto and Miyazaki in [5], and (2) 
is the geometric generalization of equations formulated by Fukumoto in [4]. See Section 2 for details.

The replacement of the sphere as a target by a more general Riemannian manifold is one of the ways to study these physical models with some geometric structures like (1) or (2). In fact, there has been many geometric analyses on the relationship between the good structure of (1) or (2) and the geometry of $N$, where $N$ is a compact Riemann surface, or more generally, a compact Kähler manifold. See, e.g., [1, 8, 9, 13] and [14].

Especially, the equation of Schrödinger map geometrically generalizes an equation proposed by Da Rios in [3]. In [1] Chang, Shatah and Uhlenbeck studied the structure of (1) with $a=b=0$. They constructed a moving frame of the induced bundle $u^{-1} T N$, and reduced the equation to a simple form of complex valued semilinear partial differential equation. More precisely, if there exists $u^{*} \in N$ such that

$$
\lim _{x \rightarrow-\infty} u(t, x)=u^{*}
$$

for any $t \in \mathbb{R},(1)$ with $a=b=0$ is reduced to

$$
q_{t}=i q_{x x}+\frac{i}{2} \kappa(u)|q|^{2} q-\frac{i}{2}\left[\int_{-\infty}^{x}|q|^{2}(\kappa(u))_{x} d x^{\prime}\right] q,
$$

where $q(t, x)$ is a complex-valued function of $(t, x)$ and $\kappa(u)$ denotes the Gaussian curvature at $u \in N$. Their idea of this reduction came from the work of Hasimoto in [7]. Their idea is also essentially related with the basic method for one-dimensional linear Schrödinger-type evolution equations. See Section 4 for details.

The purpose of this paper is to reduce the equations of dispersive flows (1) and (2) to complex valued equations by constructing a good moving frame. To state our results, we introduce Sobolev spaces $H^{\infty}(\mathbb{R})$ and $H^{\infty}(\mathbb{R} ; T N)$ defined by

$$
\begin{aligned}
& H^{\infty}(\mathbb{R})=\left\{\left.q \in C^{\infty}(\mathbb{R} ; \mathbb{C})\left|\int_{\mathbb{R}}\right| \partial_{x}^{k} q(x)\right|^{2} d x<\infty, k=0,1,2, \ldots\right\}, \\
& H^{\infty}(\mathbb{R} ; T N)=\left\{u \in C^{\infty}(\mathbb{R} ; N) \mid \int_{\mathbb{R}} g\left(\nabla_{x}^{k} u_{x}, \nabla_{x}^{k} u_{x}\right) d x<\infty, k=1,2, \ldots\right\} .
\end{aligned}
$$

For a function space $X, C(\mathbb{R} ; X)$ denotes the set of all $X$-valued continuous functions on $\mathbb{R}$. Our results are the following.

Theorem 1. Assume that the equation (1) has a solution $u \in C\left(\mathbb{R} ; H^{\infty}(\mathbb{R} ; T N)\right)$ and that $\lim _{x \rightarrow-\infty} u(t, x)=u^{*} \in N$ for any $t \in \mathbb{R}$. Then there exists a complex-valued function $q \in$ $C\left(\mathbb{R} ; H^{\infty}(\mathbb{R})\right)$ solving

$$
\begin{aligned}
q_{t}- & a q_{x x x}-i q_{x x}=\left(\frac{a}{2} \kappa(u)+2 b\right)|q|^{2} q_{x}-\left(\frac{a}{2} \kappa(u)-b\right) q^{2} \bar{q}_{x} \\
& +i a\left[\int_{-\infty}^{x}(\kappa(u))_{x} \operatorname{Im}\left(q \bar{q}_{x}\right) d x^{\prime}\right] q-\frac{i}{2}\left[\int_{-\infty}^{x}(\kappa(u))_{x}|q|^{2} d x^{\prime}\right] q+\frac{i}{2} \kappa(u)|q|^{2} q .
\end{aligned}
$$

Theorem 2. Assume that the equation (2) has a solution $u \in C\left(\mathbb{R} ; H^{\infty}(\mathbb{R} ; T N)\right)$ and that $\lim _{x \rightarrow-\infty} u(t, x)=u^{*} \in N$ for any $t \in \mathbb{R}$. Then there exists a complex-valued function $q \in$ $C\left(\mathbb{R} ; H^{\infty}(\mathbb{R})\right)$ solving

$$
\begin{aligned}
q_{t}+ & i a q_{x x x x}-i q_{x x}=i\left(b+\frac{c}{2}-\frac{a}{2} \kappa(u)\right)|q|^{2} q_{x x}+i\left(\frac{c}{2}-\frac{a}{2} \kappa(u)\right) q^{2} \bar{q}_{x x}+i\left(b+\frac{c}{2}\right) \bar{q} q_{x}^{2} \\
+ & i\left(b+\frac{3 c}{2}+\frac{a}{2} \kappa(u)\right) q\left|q_{x}\right|^{2}+\frac{i}{2} \kappa(u)|q|^{2} q+i \frac{b+c}{4} \kappa(u)|q|^{4} q \\
- & i\left[\int_{-\infty}^{x}(\kappa(u))_{x}\left\{\frac{1}{2}|q|^{2}+\frac{b+c}{4}|q|^{4}+\frac{a}{2}\left|q_{x}\right|^{2}-\frac{a}{2} q_{x x} \bar{q}-\frac{a}{2} \bar{q}_{x x} q\right\} d x^{\prime}\right] q .
\end{aligned}
$$


The meaning of our reductions is to see the essential structure of the equations (1) and (2) from a view point of the theory of linear partial differential operators. Using our reductions, one can easily understand whether the classical method of solving initial value problems works or not. See Section 4 for details. We remark that our reductions cannot be used as the tool of solving the initial value problem for the equations (1) and (2). Firstly, we impose that solutions have a fixed base point $u^{*}$ at $x=-\infty$, and such solutions are not physically interesting. Secondly, our reductions (4) and (5) contain functions depending on solutions $u$ to the original equations (1) and (2), and we need to express $u$ by $q$ to solve (4) and (5). Our reductions can be used as the tool of solving (1) and (2) only in the case that the maps are near constant and the target is the two-sphere or a hyperbolic surface.

The plan of this article is as follows. In Section 2 we derive (1) and (2) from classical mechanical models. In Section 3 we prove Theorems 1 and 2. Finally, in Section 4 we discuss our reduction from a point of view of the theory of linear dispersive partial differential equations. Throughout this paper, as has already been used in the above equation (3), (4) and (5), the partial differentiation for $\mathbb{R}$ or $\mathbb{C}$-valued functions is written by $\partial$ or the script, e.g., $\partial_{x} f, f_{x}$.

\section{Geometric generalization of physical models}

Consider the motion of a very thin isolated vortex filament in three-dimensional incompressible unbounded perfect fluid. We denote the position of the vortex filament by $\overrightarrow{\mathbf{X}}(t, x)$, where $t$ is a time, and $x$ is the arc length. Let $(\kappa, \tau)$ be the curvature and the torsion and let $\left(\overrightarrow{\mathbf{X}}_{x}, \overrightarrow{\mathbf{n}}, \overrightarrow{\mathbf{b}}\right)$ be the Frenet-Serret frame along the vortex filament $\overrightarrow{\mathbf{X}}$. In [3] Da Rios formulated the following model equation

$$
\overrightarrow{\mathbf{X}}_{t}=\kappa \overrightarrow{\mathbf{b}} .
$$

by using the so called localized induction approximation. See also [6]. In [7] Hasimoto pointed out that (6) can be formally transformed into the cubic nonlinear Schrödinger equation

$$
\psi=i \psi_{x x}+\frac{i}{2}\left[|\psi|^{2}+A(t)\right] \psi
$$

by using

$$
\psi(t, x)=\kappa(t, x) \exp \left(i \int_{0}^{x} \tau\left(t, x^{\prime}\right) d x^{\prime}\right)
$$

where $A(t)$ is a real-valued function of $t \in \mathbb{R}$. Furthermore, if we set

$$
\phi(t, x)=\psi(t, x) \exp \left(-\frac{i}{2} \int_{0}^{t} A\left(t^{\prime}\right) d t^{\prime}\right)
$$

(7) becomes

$$
\phi_{t}=i \phi_{x x}+\frac{i}{2}|\phi|^{2} \phi
$$

The correspondence $X \mapsto \psi$ or $X \mapsto \phi$ is said to be the Hasimoto transform.

Recently, other model equations of vortex filament were proposed. In [5] Fukumoto and Miyazaki proposed the following

$$
\overrightarrow{\mathbf{X}}_{t}=\kappa \overrightarrow{\mathbf{b}}+a\left[\frac{1}{2} \kappa^{2} \overrightarrow{\mathbf{X}}_{x}+\kappa_{x} \overrightarrow{\mathbf{n}}+\kappa \tau \overrightarrow{\mathbf{b}}\right]
$$


where the real constant $a \in \mathbb{R}$ is the effect of axial flow flux through the vortex tube. In [4], considering the influence of elliptical deformation of the core due to the self-induced strain, Fukumoto also proposed the following

$$
\overrightarrow{\mathbf{X}}_{t}=\kappa \overrightarrow{\mathbf{b}}+C_{1}\left[\kappa^{2} \tau \overrightarrow{\mathbf{X}}_{x}+\left(2 \kappa_{x} \tau+\kappa \tau_{x}\right) \overrightarrow{\mathbf{n}}+\left(\kappa \tau^{2}-\kappa_{x x}\right) \overrightarrow{\mathbf{b}}\right]+C_{b} \kappa^{3} \overrightarrow{\mathbf{b}}
$$

where $C_{1}, C_{b}$ are real constants.

(9) can be transformed by the Hasimoto transform into the following equation of the form

$$
\psi_{t}=i \psi_{x x}+\frac{i}{2}|\psi|^{2} \psi+a\left\{\psi_{x x x}+\frac{3}{2}|\psi|^{2} \psi_{x}\right\} .
$$

This equation (11) is called a Hirota equation, and is known as the completely integrable equation in classical mechanics. Similarly, (10) can be transformed to the following equation of the form

$$
\begin{aligned}
\psi_{t}= & i \psi_{x x}+\frac{i}{2}|\psi|^{2} \psi-i C_{1}\left\{\psi_{x x x x}+\frac{3}{2}\left(|\psi|^{2} \psi_{x x}+\psi_{x}^{2} \bar{\psi}\right)+\left(\frac{3}{8}|\psi|^{4}+\frac{1}{2}\left(|\psi|^{2}\right)_{x x}\right) \psi\right\} \\
& +i\left(C_{b}+\frac{C_{1}}{2}\right)\left\{\left(|\psi|^{2} \psi\right)_{x x}+\frac{3}{4}|\psi|^{4} \psi\right\} .
\end{aligned}
$$

We show that the equations (9) and (10) can be written in the form of equations (1) and (2), respectively. For $\vec{u}=\left(u_{1}, u_{2}, u_{3}\right) \in \mathbb{R}^{3}$ and $\vec{v}=\left(v_{1}, v_{2}, v_{3}\right) \in \mathbb{R}^{3}$, set

$$
\begin{aligned}
& (\vec{u}, \vec{v})=u_{1} v_{1}+u_{2} v_{2}+u_{3} v_{3}, \quad|\vec{u}|=\sqrt{(\vec{u}, \vec{u})}, \\
& \vec{u} \times \vec{v}=\left(u_{2} v_{3}-u_{3} v_{2}, u_{3} v_{1}-u_{1} v_{3}, u_{1} v_{2}-u_{2} v_{1}\right),
\end{aligned}
$$

and denote $\mathbb{S}^{2}=\left\{\vec{u} \in \mathbb{R}^{3}|| \vec{u} \mid=1\right\}$. If the curvature of $\overrightarrow{\mathbf{X}}$ never vanishes, that is $\overrightarrow{\mathbf{X}}_{x x} \neq \overrightarrow{0}$, the right hand side of (9) and (10) make sense and are written as

$$
\begin{aligned}
& \overrightarrow{\mathbf{X}}_{t}=\overrightarrow{\mathbf{X}}_{x} \times \overrightarrow{\mathbf{X}}_{x x}+a\left[\overrightarrow{\mathbf{X}}_{x x x}+\frac{3}{2}\left|\overrightarrow{\mathbf{X}}_{x x}\right|^{2} \overrightarrow{\mathbf{X}}_{x}\right] \\
& \overrightarrow{\mathbf{X}}_{t}=\overrightarrow{\mathbf{X}}_{x} \times \overrightarrow{\mathbf{X}}_{x x}-C_{1} \overrightarrow{\mathbf{X}}_{x} \times \overrightarrow{\mathbf{X}}_{x x x x}+C_{1} \overrightarrow{\mathbf{X}}_{x x} \times \overrightarrow{\mathbf{X}}_{x x x}+\left(C_{b}-2 C_{1}\right)\left|\overrightarrow{\mathbf{X}}_{x x}\right|^{2} \overrightarrow{\mathbf{X}}_{x} \times \overrightarrow{\mathbf{X}}_{x x},
\end{aligned}
$$

respectively. Differentiating the vortex filament equation (13) and (14) with respect to $x$, we obtain the equation for the velocity vector $\overrightarrow{\mathbf{u}}=\overrightarrow{\mathbf{X}}_{x}$ of the form

$$
\begin{aligned}
& \overrightarrow{\mathbf{u}}_{t}=\overrightarrow{\mathbf{u}} \times \overrightarrow{\mathbf{u}}_{x x}+a\left\{\overrightarrow{\mathbf{u}}_{x x x}+3\left(\overrightarrow{\mathbf{u}}_{x x}, \overrightarrow{\mathbf{u}}_{x}\right) \overrightarrow{\mathbf{u}}+\frac{3}{2}\left|\overrightarrow{\mathbf{u}}_{x}\right|^{2} \overrightarrow{\mathbf{u}}_{x}\right\} \\
& \overrightarrow{\mathbf{u}}_{t}=\overrightarrow{\mathbf{u}} \times \overrightarrow{\mathbf{u}}_{x x}-C_{1} \overrightarrow{\mathbf{u}} \times \overrightarrow{\mathbf{u}}_{x x x x}+\left(C_{b}-2 C_{1}\right)\left(\left|\overrightarrow{\mathbf{u}}_{x}\right|^{2} \overrightarrow{\mathbf{u}} \times \overrightarrow{\mathbf{u}}_{x}\right)_{x},
\end{aligned}
$$

respectively. Since $x$ is the arc length, $|\overrightarrow{\mathbf{u}}|=1$, which implies that $\overrightarrow{\mathbf{u}}$ lies on $\mathbb{S}^{2}$.

Here assume that $u(t, x) \in \mathbb{S}^{2}$ for any $(t, x)$. Let $V$ be any vector field along $u$, namely, $V(t, x) \in T_{u} \mathbb{S}^{2}$ for any $(t, x)$. Regarding $\mathbb{S}^{2}$ as the two-sphere with the standard metric induced from $\mathbb{R}^{3}$, we see from the definition of the covariant derivative on $\mathbb{S}^{2}$ that

$$
\nabla_{x} V=V_{x}-\left(V_{x}, u\right) u=V_{x}+\left(V, u_{x}\right) u .
$$

Thus we have

$$
\begin{aligned}
& \nabla_{x} u_{x}=u_{x x}+\left|u_{x}\right|^{2} u, \\
& \nabla_{x}^{2} u_{x}=u_{x x x}+3\left(u_{x x}, u_{x}\right) u+\left|u_{x}\right|^{2} u_{x}, \\
& \nabla_{x}^{3} u_{x}=u_{x x x x}+4\left(u_{x x x}, u_{x}\right) u+3\left|u_{x x}\right|^{2} u+5\left(u_{x x}, u_{x}\right) u_{x}+\left|u_{x}\right|^{2} u_{x x}+\left|u_{x}\right|^{4} u .
\end{aligned}
$$


Also, the operation $u \times V$, rotating $V$ by $\pi / 2$ degrees for any $V \in T_{u} \mathbb{S}^{2}$, acts as a complex number in the tangent space. Thus $J_{u} V=u \times V$ for any vector field $V$ along $u$, which implies

$$
u \times u_{x x}=u \times \nabla_{x} u_{x}=J_{u} \nabla_{x} u_{x} .
$$

Noting these relations, we see that (15) is written as (1) with $b=a / 2$ and (16) is written as (2) with $a=C_{1}, b=C_{b}-C_{1}, c=2 C_{b}+C_{1}$ respectively.

\section{Proofs}

In this section, we prove Theorem 1 and 2 proceeding as in [1].

Proof of Theorem 1. Assume that there exists a point $u^{*} \in N$ and that equation (1) has a smooth solution on $\mathbb{R} \times \mathbb{R}$ such that $u(t, x) \rightarrow u^{*} \in N$ as $x \rightarrow-\infty$. Let $\{e, J e\}$ denote the orthonormal frame for $u^{-1} T N$ constructed in the following manner:

Fix a unit vector $e_{0} \in T_{u^{*}} N$, namely, $g_{u^{*}}\left(e_{0}, e_{0}\right)=1$. And for any $t \in \mathbb{R}$, let $e(t, x) \in T_{u(t, x)} N$ be the parallel translation of $e_{0}$ along the curve $u(t, \cdot)$, that is,

$$
\begin{aligned}
& \nabla_{x} e(t, x)=0, \\
& \lim _{x \rightarrow-\infty} e(t, x)=e_{0} .
\end{aligned}
$$

In local coordinate $\left(U, \phi=\left(u^{1}, u^{2}\right)\right)$ around $u(t, x)$, where the metric is given by $g=\lambda(z, \bar{z}) d z d \bar{z}$ with a complex coordinate $z=u^{1}+i u^{2}$, set

$$
e(t, x)=e^{1}(t, x)\left(\frac{\partial}{\partial u^{1}}\right)_{u}+e^{2}(t, x)\left(\frac{\partial}{\partial u^{2}}\right)_{u}, \quad \text { and } \quad \zeta=e^{1}+i e^{2} .
$$

Here $\left\{\left(\frac{\partial}{\partial u^{1}}\right)_{u},\left(\frac{\partial}{\partial u^{2}}\right)_{u}\right\}$ is the standard basis on $T_{u} N$. Then it follows from (17) that, $\zeta$ satisfies

$$
\zeta_{x}+2(\log \lambda)_{z} z_{x} \zeta=0
$$

The equation (19) is a linear first-order ordinary differential equation, and (17)-(18) has a global solution $e$.

Since $\{e, J e\}$ is an orthonormal frame along $u$, we have

$$
0=\partial_{t}\left[g_{u}(e, e)\right]=2 g_{u}\left(\nabla_{t} e, e\right),
$$

which implies

$$
\nabla_{t} e=\alpha J e,
$$

for some real-valued function $\alpha(t, x)$. Moreover, in this frame the coordinates of $u_{t}$ and $u_{x}$ are given by two complex valued functions $p$ and $q$, namely, set

$$
\begin{aligned}
& u_{t}=p_{1} e+p_{2} J e, \\
& u_{x}=q_{1} e+q_{2} J e,
\end{aligned}
$$

where $p_{1}, p_{2}, q_{1}, q_{2}$ are real-valued functions of $(t, x)$, and set

$$
p=p_{1}+i p_{2}, \quad \text { and } \quad q=q_{1}+i q_{2} .
$$

The relationships between $p, q$ and $\alpha$ are as follows. Using the equation (1), we have

$$
p=a q_{x x}+i q_{x}+b|q|^{2} q .
$$


The compatibility condition $\nabla_{x} u_{t}=\nabla_{t} u_{x}$ implies

$$
p_{x}=q_{t}+i \alpha q \text {. }
$$

Combining (23) and (24), we have

$$
q_{t}=a q_{x x x}+i q_{x x}-i \alpha q+b\left(|q|^{2} q\right)_{x} .
$$

Moreover, we can obtain

$$
\alpha_{x}=-\kappa(u) \operatorname{Im}(\bar{q} p),
$$

where $\kappa(u)=g_{u}(R(e, J e) J e, e)$ is the Gaussian curvature of $N$ at $u$, and $R$ is the Riemann curvature tensor. Indeed, on one hand, using (17), (20) and the identity $\nabla_{t} \nabla_{x} e-\nabla_{x} \nabla_{t} e=$ $R\left(u_{t}, u_{x}\right) e$, we have

$$
R\left(u_{t}, u_{x}\right) e=-\nabla_{x}(\alpha J e)=-\alpha_{x} J e .
$$

On the other hand, combining (21), (22) and the identity $R(X, Y) e=-R(Y, X) e$ for any vector fields $X$ and $Y$ along $u$, we have

$$
R\left(u_{t}, u_{x}\right) e=\left(p_{2} q_{1}-p_{1} q_{2}\right) R(J e, e) e=\operatorname{Im}(\bar{q} p) R(J e, e) e .
$$

Hence it follows that

$$
-\alpha_{x} J e=\operatorname{Im}(\bar{q} p) R(J e, e) e .
$$

Thus taking the inner product of (27) and $-J e$, we have (26).

Substituting (23) into (26), we have

$$
\alpha_{x}=\kappa(u)\left(-\frac{1}{2}|q|^{2}+a \operatorname{Im}\left(q \bar{q}_{x}\right)\right)_{x} .
$$

Integrating this over $(-\infty, x]$, we get

$$
\begin{aligned}
\alpha(t, x)= & \int_{-\infty}^{x} \kappa(u)\left(t, x^{\prime}\right)\left(-\frac{1}{2}|q|^{2}+a \operatorname{Im}\left(q \bar{q}_{x}\right)\right)_{x}\left(t, x^{\prime}\right) d x^{\prime}+\alpha(t,-\infty) \\
= & \kappa(u)(t, x)\left(-\frac{1}{2}|q|^{2}+a \operatorname{Im}\left(q \bar{q}_{x}\right)\right)(t, x)+\frac{1}{2} \int_{-\infty}^{x}(\kappa(u))_{x}\left(t, x^{\prime}\right)\left|q\left(t, x^{\prime}\right)\right|^{2} d x^{\prime} \\
& -a \int_{-\infty}^{x}(\kappa(u))_{x}\left(t, x^{\prime}\right) \operatorname{Im}\left(q \bar{q}_{x}\right)\left(t, x^{\prime}\right) d x^{\prime}+A(t),
\end{aligned}
$$

where $A(t)=\alpha(t,-\infty)$. Substituting (28) into (25), we have

$$
\begin{aligned}
q_{t}- & a q_{x x x}-i q_{x x}=\left(\frac{a}{2} \kappa(u)+2 b\right)|q|^{2} q_{x}-\left(\frac{a}{2} \kappa(u)-b\right) q^{2} \bar{q}_{x} \\
+ & i a\left[\int_{-\infty}^{x}(\kappa(u))_{x} \operatorname{Im}\left(q \bar{q}_{x}\right) d x^{\prime}\right] q-\frac{i}{2}\left[\int_{-\infty}^{x}(\kappa(u))_{x}|q|^{2} d x^{\prime}\right] q+\frac{i}{2} \kappa(u)|q|^{2} q-i A(t) q .
\end{aligned}
$$

If we set

$$
Q(t, x)=q(t, x) \exp \left(i \int_{0}^{t} A(\tau) d \tau\right)
$$

$Q(t, x)$ solves (4). This completes the proof of Theorem 1 . 
Proof of Theorem 2. In the same way as the previous proof, construct the moving frame $\{e, J e\}$ and set complex-valued functions $p$ and $q$.

The relationships between $p, q$ and $\alpha$ are as follows. The equation (2) shows that

$$
-i p=q_{x}-a q_{x x x}+b|q|^{2} q_{x}+\frac{c}{2}\left(|q|^{2}\right)_{x} q
$$

Using $\nabla_{x} u_{t}=\nabla_{t} u_{x}$, we have

$$
p_{x}=q_{t}+i \alpha q
$$

Combining (30) and (31), we have

$$
-i q_{t}=-a q_{x x x x}+q_{x x}+b\left(|q|^{2} q_{x}\right)_{x}+\frac{c}{2}\left(\left(|q|^{2}\right)_{x} q\right)_{x}-\alpha q .
$$

Using the curvature tensor $\nabla_{t} \nabla_{x} e-\nabla_{x} \nabla_{t} e=R\left(u_{t}, u_{x}\right) e$, we have

$$
\alpha_{x}=-\kappa(u) \operatorname{Im}(\bar{q} p),
$$

where $\kappa(u)=g_{u}(R(e, J e) J e, e)$ is the Gaussian curvature of $N$ at $u$.

Substituting (23) into (33), we have

$$
\alpha_{x}=-\kappa(u)\left(\frac{1}{2}|q|^{2}+\frac{b+c}{4}|q|^{4}\right)_{x}+\frac{a}{2} \kappa(u)\left(\bar{q}_{x x x} q+q_{x x x} \bar{q}\right)
$$

Substituting the integration of the right hand side of $(34)$ on $(-\infty, x]$ into $(32)$, we have

$$
\begin{aligned}
& q_{t}+i a q_{x x x x}-i q_{x x}=i b\left(|q|^{2} q_{x}\right)_{x}+i \frac{c}{2}\left\{\left(|q|^{2}\right)_{x} q\right\}_{x}-i A(t) q \\
& \quad-i q \int_{-\infty}^{x}\left\{-\kappa(u)\left(\frac{1}{2}|q|^{2}+\frac{b+c}{4}|q|^{4}\right)_{x}+\frac{a}{2} \kappa(u)\left(\bar{q}_{x x x} q+q_{x x x} \bar{q}\right)\right\} d x^{\prime} .
\end{aligned}
$$

Calculation of the fourth terms of the right hand side of (35) by the use of integrations by parts implies that

$$
\begin{aligned}
q_{t}+ & i a q_{x x x x}-i q_{x x}=i\left(b+\frac{c}{2}-\frac{a}{2} \kappa(u)\right)|q|^{2} q_{x x}+i\left(\frac{c}{2}-\frac{a}{2} \kappa(u)\right) q^{2} \bar{q}_{x x}+i\left(b+\frac{c}{2}\right) \bar{q} q_{x}^{2} \\
+ & i\left(b+\frac{3 c}{2}+\frac{a}{2} \kappa(u)\right) q\left|q_{x}\right|^{2}+\frac{i}{2} \kappa(u)|q|^{2} q+i \frac{b+c}{4} \kappa(u)|q|^{4} q \\
- & i\left[\int_{-\infty}^{x}(\kappa(u))_{x}\left\{\frac{1}{2}|q|^{2}+\frac{b+c}{4}|q|^{4}+\frac{a}{2}\left|q_{x}\right|^{2}-\frac{a}{2} q_{x x} \bar{q}-\frac{a}{2} \bar{q}_{x x} q\right\} d x^{\prime}\right] q-i A(t) q .
\end{aligned}
$$

We can take $A(t) \equiv 0$ without loss of generality by the same way as we use (29) in the previous proof. That is, if we set

$$
Q(t, x)=q(t, x) \exp \left(i \int_{0}^{t} A(\tau) d \tau\right)
$$

it is easy to check that $Q$ solves (5). Thus we complete the proof. 


\section{Concluding remarks}

Our reduction via moving frame leads to an understanding of the essential structure of the differential equations (1) and (2). Unfortunately, however, the results of this paper alone are not sufficient for establishing well-posedness of the initial-value problem. In this section we discuss the relationship between our reduction and the theory of linear partial differential operators. One can consult with [10] on the basic theory of linear dispersive partial differential operators.

Here we review the theory of one-dimensional linear dispersive partial differential operators. Consider the initial value problem for one-dimensional Schrödinger-type evolution equations of the form

$$
\begin{aligned}
& v_{t}-i v_{x x}+b(x) v_{x}+c(x) v=f(t, x) \quad \text { in } \quad \mathbb{R} \times \mathbb{R}, \\
& v(0, x)=v_{0}(x) \quad \text { in } \mathbb{R},
\end{aligned}
$$

where $b(x), c(x) \in \mathscr{B}^{\infty}(\mathbb{R})$, which is the set of all smooth functions on $\mathbb{R}$ whose derivative of any order are bounded on $\mathbb{R}, v(t, x)$ is a complex-valued unknown function, and $f(t, x)$ and $v_{0}(x)$ are given functions. It is well-known that the initial value problem (36)-(37) is well-posed in the framework of $L^{2}(\mathbb{R})$ if and only if

$$
\sup _{x \in \mathbb{R}}\left|\int_{0}^{x} \operatorname{Im} b(y) d y\right|<\infty .
$$

See [10] for details. This condition describes the balance of the local smoothing effect of $\exp \left(i t \partial_{x}^{2}\right)$

$$
\left\|\left(1+x^{2}\right)^{-\delta}\left(-\partial_{x}^{2}\right)^{1 / 4} e^{i t \partial_{x}^{2}} \phi\right\|_{L^{2}\left(\mathbb{R}^{2}\right)} \leqslant C\|\phi\|_{L^{2}(\mathbb{R})}, \quad \delta>1 / 4,
$$

and the strength of the bad part of the first order term $i \operatorname{Im}\{b(x)\} \partial_{x}$. See, e.g., $[2,15]$ and [20] for the local smoothing effect of linear dispersive partial differential equations with constant coefficients. If the condition (38) breaks down, the equation (36) behaves like the CauchyRiemann equation $v_{i}+i v_{x}=0$ and the initial value problem (36)-(37) can be solvable only in some framework of real-analytic functions. Under the condition (38), a gauge transform

$$
v(t, x) \longmapsto w(t, x)=v(t, x) \exp \left(\frac{i}{2} \int_{0}^{x} b(y) d y\right)
$$

is automorphic on $C\left(\mathbb{R} ; L^{2}(\mathbb{R})\right)$, and (36)-(37) becomes the initial value problem of the form

$$
w_{t}-i w_{x x}+d(x) w=g(t, x), \quad w(0, x)=w_{0}(x),
$$

which is easy to solve. We remark that if we replace $x \in \mathbb{R}$ by $x \in \mathbb{R} / \mathbb{Z}$, then the local smoothing effect breaks down and the condition (38) becomes

$$
\int_{0}^{1} \operatorname{Im} b(y) d y=0 .
$$

The generalized Hasimoto transform introduced in [1] corresponds to (39). The equation (1) with $a=b=0$ behaves like symmetric hyperbolic systems since $\nabla^{N} J=0$, where $\nabla^{N}$ is the Levi-Civita connection of $(N, J, g)$. This fact is implicitly applied to proving of the existence of solutions to the initial value problem. See [8, 9, 14] and [16]. From a point of view of the theory of linear partial differential operators, this is due to the fact that the $L^{2}$-norm given by the Kähler metric of the form

$$
\left\{\int_{\mathbb{R}} g(V, V) d x\right\}^{1 / 2} \quad \text { for } \quad V \in \Gamma_{0}\left(u^{-1} T N\right),
$$


corresponds to the modified norm

$$
\left\{\int_{\mathbb{R}}|v|^{2} \exp \left(-\int_{0}^{x} \operatorname{Im} b(y) d y\right) d x\right\}^{1 / 2}
$$

for (36). Here $\Gamma_{0}\left(u^{-1} T N\right)$ is the set of compactly supported smooth sections of $u^{-1} T N$.

Similarly, we discuss one-dimensional third and fourth-order linear dispersive partial differential equations of the forms

$$
\begin{aligned}
& v_{t}+v_{x x x}+\alpha(x) v_{x x}+\beta(x) v_{x}+\gamma(x) v=0, \\
& v_{t}+v_{x x x x}+A(x) v_{x x x}+B(x) v_{x x}+C(x) v_{x}+D(x) v=0,
\end{aligned}
$$

where $v: \mathbb{R} \times \mathbb{R} \rightarrow \mathbb{C}$ is an unknown function, and $\alpha, \beta, \gamma, A, B, C, D \in \mathscr{B}^{\infty}(\mathbb{R})$. Tarama studied the initial value problem for (42) in [18] and [19], and Mizuhara studied the initial value problem for (43) in [11]. The initial value problem for (42) is $L^{2}$-well-posed if and only if

$$
\begin{aligned}
& \sup _{x \in \mathbb{R}}\left|\int_{0}^{x} \operatorname{Re} \alpha(s) d s\right|<\infty, \\
& \sup _{\substack{(x, y) \in \mathbb{R}^{2} \\
x \neq y}}\left|\int_{y}^{x} \operatorname{Im}\left\{\beta(s)+\frac{\alpha(s)^{2}}{3}\right\} d s\right||x-y|^{-1 / 2}<\infty .
\end{aligned}
$$

Under the conditions (44) and (45), the second order term in (42) is eliminated by a gauge transform

$$
v(t, x) \longmapsto w(t, x)=v(t, x) \exp \left(\frac{1}{3} \int_{0}^{x} \alpha(y) d y\right),
$$

and the bad part of the first order term of (42) is canceled out by a transform defined by a pseudodifferential operator. See [18] and [19] for details. When $x \in \mathbb{R} / \mathbb{Z}$, (44) and (45) become

$$
\int_{0}^{1} \operatorname{Re} \alpha(y) d y=\int_{0}^{1} \operatorname{Im}\left\{\beta(y)+\frac{\alpha(y)^{2}}{3}\right\} d y=0 .
$$

The generalized Hasimoto transform in the proof of Theorem 1 corresponds to (46). In [13] the author proved local and global existence theorems of the initial value problem for (1) on $\mathbb{R} \times \mathbb{R} / \mathbb{Z}$ with values in a Kähler manifold. He made use of the classical energy estimates of (41). Notice that (1) also behaves like symmetric hyperbolic systems since $\nabla^{N} g=0$ and $\nabla^{N} J=0$. The $L^{2}$-norm (41) works as if the unknown functions were transformed by (46) and the pseudodifferential operator. In other words, if one see the metric tensor $g$ as a real symmetric matrix or a hermitian matrix, a commutator $\left[g, I \partial_{x x x}+J \partial_{x x}\right]$ eliminates the bad part of the second and the first order terms, where $I$ is the identity matrix. It is worth to mention that Nishiyama and Tani studied existence theorems of the initial value problem for the third-order two-sphere valued model (15) in [12] and [17].

The necessary and sufficient condition of the $L^{2}$-well-posedness of the initial value problem for (43) was given in [11] under some technical condition. One cannot expect the existence of a smooth closed curve, that is a periodic solution in $x$, since the classical energy method breaks down for $(2)$.

\section{Acknowledgements}

The author expresses gratitude to Hiroyuki Chihara for several discussions and valuable advice. Also, thanks to the referees for carefully reading the manuscript. The author is supported by the JSPS Research Fellowships for Young Scientists and the JSPS Grant-in-Aid for Scientific Research No.19·3304. 


\section{References}

[1] Chang N.H., Shatah J., Uhlenbeck K., Schrödinger maps, Comm. Pure Appl. Math. 53 (2000), 590-602.

[2] Constantin P., Saut J.C., Local smoothing properties of dispersive equations, J. Amer. Math. Soc. 1 (1989), 413-446.

[3] Da Rios, On the motion of an unbounded fluid with a vortex filament of any shape, Rend. Circ. Mat. Palermo 22 (1906), 117-135 (in Italian).

[4] Fukumoto Y., Motion of a curved vortex filament: higher-order asymptotics, in Proc. of IUTAM Symposium on Geometry and Statistics of Turbulence (1999, Hayama), Editors T. Kambe, T. Nakano and T. Miyauchi, Fluid Mech. Appl., Vol. 59, Kluwer Acad. Publ., Dordrecht, 2001, 211-216.

[5] Fukumoto Y., Miyazaki T., Three-dimensional distortions of a vortex filament with axial velocity, J. Fluid Mech. 222 (1991), 369-416.

[6] Hama F.R., Progressive deformation of a curved vortex filament by its own induction, Phys. Fluids 5 (1962), 1156-1162.

[7] Hasimoto H., A soliton on a vortex filament, J. Fluid. Mech. 51 (1972), 477-485.

[8] Koiso N., The vortex filament equation and a semilinear Schrödinger equation in a Hermitian symmetric space, Osaka J. Math. 34 (1997), 199-214.

[9] Koiso N., Vortex filament equation and semilinear Schrödinger equation, in Nonlinear Waves (1995, Sapporo), Gakuto Intern. Ser. Math. Sci. Appl., Vol. 10, 1997, 231-236.

[10] Mizohata M., On the Cauchy problem, Academic Press, 1985.

[11] Mizuhara R., The initial value problem for third and fourth order dispersive equations in one space dimensions, Funkcial. Ekvac. 49 (2006), 1-38.

[12] Nishiyama T., Tani A., Initial and initial-boundary value problems for a vortex filament with or without axial flow, SIAM J. Math. Anal. 27 (1996), 1015-1023.

[13] Onodera E., A third-order dispersive flow for closed curves into Kähler manifolds, J. Geom. Anal. 18 (2008), to appear, arXiv:0707.2660.

[14] Pang P.Y.Y., Wang H.Y., Wang Y.D., Schrödinger flow on Hermitian locally symmetric spaces, Comm. Anal. Geom. 10 (2002), 653-681.

[15] Sjölin P., Regularity of solutions to the Schrödinger equation, Duke Math. J. 55 (1987), 699-715.

[16] Sulem P.-L., Sulem C., Bardos C., On the continuous limit for a system of classical spins, Comm. Math. Phys. 107 (1986), 431-454.

[17] Tani A., Nishiyama T., Solvability of equations for motion of a vortex filament with or without axial flow, Publ. Res. Inst. Math. Sci. 33 (1997), 509-526.

[18] Tarama S., On the wellposed Cauchy problem for some dispersive equations, J. Math. Soc. Japan 47 (1995), $143-158$.

[19] Tarama S., Remarks on $L^{2}$-wellposed Cauchy problem for some dispersive equations, J. Math. Kyoto Univ. 37 (1997), 757-765.

[20] Vega L., The Schroödinger equation: pointwise convergence to the initial date, Proc. Amer. Math. Soc. 102 (1988), 874-878. 